\title{
Live-Imaging of Myelin in Animal Models and in Human
}

\author{
Bernard Zalc* \\ Sorbonne Universités, UPMC Univ Paris, CNRS, INSERM, Institut du Cerveau et de la Moelle épinière (ICM), \\ GH Pitié-Salpêtrière Paris, France
}

The pioneer work of Paul Broca (1824-1880) was the first demonstration that brain is functionally not homogeneous, but on the contrary constituted by the assembly of different areas each responsible for specific function. Investigating aphasic patients Broca described a small area in the left frontal lobe, which he described as responsible for articulate language $[1,2]$. This region is now known as Broca's area. Since then the localizationism theory has extended and in association to the long-life concept that only neurons were true functional cells, it has generally been assumed that each brain function is driven by some groups or subpopulation of neurons. As a consequence, any neurological dysfunction had to be attributed to the lesion of a subgroup of neuronal cell. One of the most puzzling situation had been reached by Gerstmann's syndrome, a condition where a small lesion localized in the dominant inferior parietal lobe results in the association of four apparently completely unrelated symptoms: dysgraphia/agraphia dyscalculia/acalculia, finger agnosia, and left-right disorientation. First described in 1924 by Joseph Gerstmann $[3,4]$ this syndrome has stirred a controversy for over 80 years among neurologists questioning which population of cortical neurons localized in the angular and supramarginal gyrus could be responsible for such a diversity of cognitive functions. It is only very recently with the development of

${ }^{*}$ Correspondence to: Bernard Zalc, Sorbonne Universités, UPMC Univ Paris 6, CNRS, INSERM, Institut du Cerveau et de la Moelle épinière (ICM), GH Pitié-Salpêtrière F-75013 Paris, France. diffusion tensor technique associated with functional MRI that Rusconi and colleagues have succeeded to solve this enigma showing that a pure form of Gerstmann's syndrome might arise from disconnection, via a lesion, to separate but co-localized myelinated fiber tracts in the parietal lobe [5]. This has been an exemplary demonstration on how structural and functional connectivity studies contribute to improve the understanding of cognitive deficits. It is of interest to note that recent reinvestigation by magnetic resonance of the brain of Louis Victor Leborgne, Broca's first patient, (also known as «Tan-Tan » since this was the only syllable the patient could articulate), has shown disruption extended to connections projecting to areas distant from the lesion, illustrating that the damaged tract were linking areas functionally engaged for tasks related to language production [6]. Altogether these examples demonstrate the crucial importance of visualization of myelinated tracts. The aim of this special issue of "Brain Plasticity" is to collect contributions both in animal models as well as in human on how to investigate in vivo white matter tracts. Je Bin and David Lyons illustrate the immense advantage of combining fluorescent reporter genetically introduced into transparent vertebrates, zebra fish and xenopus tadpoles. Geneviève Rougon, Sophie Brasselet and Franck Debarbieux address the recent development of intravital imaging techniques in rodent, with a special focus on observation of inflammatory response and demyelinating lesions accompanied by axonal degeneration during Experimental Autoimmune Encephalomyelitis. 
In human, myelination begins during fetal development. Jessica Dubois and colleagues illustrate how the recent use of non-invasive techniques such as magnetic resonance imaging (MRI) and magnetoand electroencephalography (M/EEG) has opened the possibility to link the progressive acquisition of sensorimotor and cognitive functions with myelination of fiber tracts transferring and processing information between different brain regions. In contrast to any other membrane the lipid/protein ratio of myelin is 70/30, i.e., an inverted ratio in comparison with other biological membranes. As a consequence it is estimated that $40 \%$ of the mass of myelin is compartmentalized water. Conventional MRI is based on the resonance of proton, and the majority of the signal measured by magnetic resonance (MR) emanates from hydrogen in water molecules. Although white matter tracts may therefore appear not ideal structures to be visualized by conventional MRI, Cornelia Laule and Alex MacKay show how more sophisticated treatment of MR signal allows measuring the water content of myelin. In their review, they illustrate how myelin content imaging can be used to study animal models and different human neurological conditions. To have access to direct measurement of myelin Benedetta Bodini and Bruno Stankoff have developed different ligands, which bind to myelin components. After labeling with a positron emitter, such myelin ligands allow direct visualization of myelinated tracts and quantification of myelin content using Positron
Emission Tomography (PET). Finally, Hughes Duffau reviews original data obtained during neurosurgical intervention on human by direct axonal electrostimulation of myelinated tracts in adults, with regard to the functional connectivity mediating the sensorimotor, visuo-spatial, language, cognitive and emotional functions, as well as the interactions between these different sub-networks, leading ultimately to explore consciousness.

\section{REFERENCES}

[1] Broca P. Perte de la parole, ramollissement chronique et destruction partielle du lobe antérieur gauche. Bulletin de la société française d'anthropologie. Séance du 18 avril 1861, t. 2, p. 235-238;

[2] Broca P. Localisations des fonctions cérébrales. Siège de la faculté du langage articulé. Bulletin de la société française d'anthropologie. Séance du 16 avril 1863, t. 4, p. 200-204.

[3] Gerstmann J. Fingeragnosie. Eine umschriebene Störung der Orientierung am eigenen Körper. Wien Klin Wochenschr. 1924;40:1010-2.

[4] Gerstmann J. Fingeragnosie und Agraphie-ein neues Syndrom. Arch Psychiatr Nervenkr Z Gesamte Neurol Psychiatr. 1927;108:152-77.

[5] Rusconi E, Pinel P, Dehaene S, Kleinschmidt A. The enigma of Gerstmann's syndrome revisited: A telling tale of the vicissitudes of neuropsychology. Brain. 2010;133(Pt 2):320-32. doi: 10.1093/brain/awp281. Review.

[6] Catani M, Mesulam MM, Jakobsen E, Malik F, Martersteck A, Wieneke C, Thompson CK, Thiebaut de Schotten M, Dell'Acqua F, Weintraub S, Rogalski E. A novel frontal pathway underlies verbal fluency in primary progressive aphasia. Brain. 2013;136(Pt 8):2619-28. doi: 10.1093/brain/awt163 